\title{
Tactile letter recognition: Pattern duration and modes of pattern generation
}

\author{
JAMES C. CRAIG \\ Indiana University, Bloomington, Indiana 47405
}

\begin{abstract}
Measurements were made of the ability of subjects to identify vibrotactile patterns presented to their fingertips. The patterns were letters of the alphabet generated on the tactile display of the Optacon. Five different modes of pattern generation were examined. Two of the modes, static and scan, involved full-field presentations of the letters. In the remaining three modes, patterns were generated by presenting parts of the letters sequentially. In one mode, the letters were exposed by a slit passing across them. In the other two modes, the patterns were generated as though the letter were being drawn on the skin. Performance in all five modes was examined as a function of pattern duration, with durations ranging from 4 to $1,000 \mathrm{msec}$. Increasing duration, up to $400 \mathrm{msec}$, resulted in generally improved performance, although the functions relating performance and duration differed according to the mode of presentation. Contrary to previous results, the static mode produced the best overall performance level. Some possible reasons for the disagreement between the present results and previous results and some models of cutaneous pattern recognition are discussed.
\end{abstract}

Loomis, in the course of several studies of tactile pattern perception (Apkarian-Stielau \& Loomis, 1975; Loomis, 1974; Loomis \& Apkarian-Stielau, 1976), has developed a theory of cutaneous form perception that maintains that the skin behaves as though a low-pass spatial filter were interposed somewhere between the stimulus and the perception of that stimulus. The reason that certain patterns cannot be discriminated from one another, according to this view, is that these patterns differ from one another primarily on the basis of their higher spatial-frequency components. If the skin cannot resolve these spatial frequencies, the two patterns are considered to be identical. For example, discriminating the difference between " $O$ " and " $Q$," a discrimination that involves resolving higher spatial frequencies, should be difficult, whereas discriminating between " $T$ " and " $\mathrm{L}$," a discrimination that involves primarily lower spatial frequencies, should be relatively easy.

Support for this theoretical position came from an examination of several modes of generating vibrotactile patterns. In one study, Loomis (1974) presented letters of the alphabet to subjects using the TVSS, a device that converts optical patterns to patterns of vibration using a $20 \times 20$ array of vibrators placed against a subject's back (Bach-y-Rita, 1972; White, Saunders, Scadden, Bach-y-Rita, \& Collins, 1970). Four modes of pattern generation were compared. Two of the modes involved full-field presentations: in one mode the letter was presented statically centered on the array and in the other, a scan

This research was supported by Grant NS-09783 from the National Institutes of Health. mode, the letter moved across the array. In the other two modes, only a portion of the letter was exposed at a time through a slit. In one of these slit modes, a vertical slit moved over a stationary letter, and in the other, the letter moved behind a stationary slit. The two full-field modes produced poorer letter recognition than the two slit-scan modes. Loomis attributed this difference to the fact that the two fullfield modes require an identification to be made solely on the basis of spatial-frequency analysis, whereas the slit modes contain phase information as well. Loomis conducted some analogous experiments with blurred visual stimuli, in which the higher spatial frequencies are lost, and found a similar pattern of results (Loomis \& Apkarian-Stielau, 1976).

Loomis predicted that very good performance would be obtained tactually by a sequential presentation of a pattern, a mode similar to the children's game of backwriting. Such a mode was found, by Beauchamp, Matheson, and Scadden (1971) using the TVSS, to be better than a full-field scan mode. Loomis's theory predicts that the sequential mode would be superior to other modes because "one would expect letter recognition to be limited more by localization acuity than by spatial resolution. This follows because phase information in the lower frequencies, varying as a function of time, would carry the identity of the letter"' (Loomis, 1974, p. 405).

The experiments testing Loomis's theory were carried out on a large area of skin, the back, and the duration of the patterns was relatively long, greater than $1 \mathrm{sec}$. Sherrick (1979) reported the results of a study using a more compact array on the thigh and presenting patterns for a briefer period of time, 
300 msec. The task in the study was to indicate the orientation of a line, with a number of different modes of generating patterns being tested, including static, scan, and slit-scan. The results did not parallel the Loomis results, the scan mode being noticeably inferior to the other modes.

In a recent study, Craig (1980) compared two modes of pattern generation, static and scan, in a letterrecognition task. In view of the commonly held ideas about the importance of movement in cutaneous pattern perception, the results were surprising, in that the static mode produced generally better performance than the scan mode. More important for the present discussion of Loomis's theory is that varying pattern duration produced marked differences in performance and that performance in the two modes changed differentially as a function of duration. This result indicates that comparisons among modes at a single duration may produce a particular rank ordering of performances but that testing at another duration may produce a different rank ordering. There is, however, nothing in the ideas of limited spatial resolution, as currently formulated by Loomis, to predict changes in rank ordering among modes as a function of duration.

The topic of modes of vibrotactile pattern generation and the effect that changes in duration have on these modes is relevant, not only to theoretical accounts of cutaneous pattern recognition, but also to the design and use of cutaneous communication devices. These devices are used to present information to the sensorially handicapped. The duration of patterns generated with such devices varies with the type of device, kind of information being processed, or user capability. With the Optacon, for example, reading rates for individuals vary from 10 to 100 words/min (Craig, 1977; Goldish \& Taylor, 1974). If the relative effectiveness of different modes changes as a function of duration, designers of these displays might want to incorporate several modes of pattern generation in their tactile displays, with different modes being used for different rates of information transmission.

\section{METHOD}

\section{Subjects}

The subjects were hourly employees of the laboratory. Four women were tested in the first set of measurements. Three women and one man were tested in the second set of measurements. All subjects passed a screening test with the Optacon prior to their being employed. The selection procedure is described in Craig $(1978,1980)$. Prior to data collection, the subjects received practice with all modes on which they were to be tested.

\section{Apparatus}

The apparatus has been described previously (Craig, 1980). Briefly, it consisted of an Optacon interfaced with a PDP-11/34 computer. The tactile array of the Optacon, measuring $1.1 \times$ $2.7 \mathrm{~cm}$, consists of a $6 \times 24$ array of pins. The computer permits any pin in the 144-element array to be addressed and made to vibrate at $230 \mathrm{~Hz}$.

\section{Procedure}

The patterns generated were sans-serif, uppercase letters of the alphabet. The letters, generated on the Optacon display, occupied the top 18 rows and all 6 columns of the display, with two exceptions: the letter "I" occupied only one column and the letter " $J$ " occupied five columns. The area of the display occupied by most letters measured $1.1 \times 2.0 \mathrm{~cm}$. The amplitude of vibration was set at a comfortable level, a level that remained unchanged across subjects and modes.

A subject was seated in front of the Optacon with his/her left index finger resting on the tactile array. The subject wore earphones through which white noise was presented to reduce auditory cues and distractions. To begin a trial, the subject pressed a key on a keyboard. The subject then received a cue stimulus (the letter "I" presented in the static mode for $50 \mathrm{msec}$ in the first set of measurements and for $12 \mathrm{msec}$ in the second set of measurements), followed $1 \mathrm{sec}$ later by a single uppercase letter. The subject responded by pressing one of the keys on the keyboard and received feedback in the form of the correct letter's appearing on a visual monitor.

Two sets of measurements were made. In the first set, four modes were tested. The modes were static, scan, slit-scan, and continuous sequential. In the static mode ( $\mathrm{St}$ ), all elements making up the letters were turned on and off together. In the scan mode (Sc), the usual way in which the Optacon is used, the letters passed across the array from right to left. In the slit-stan mode (SS), the letter was exposed, two columns at a time, by a slit passing from right to left across the array. In this slit-scan mode, the letter was stationary on the array and the slit passed across the letter, revealing it column by column.

To generate patterns in the continuous sequential mode (CS), each letter was divided into six parts. As far as possible, the parts were composed of approximately equal numbers of pins, maintaining the natural strokes that would be made in printing the letter. The parts were activated in a sequence that would be followed in the normal course of the printing of the letter. The perceptual effect produced by this mode was that of a letter being lightly and rapidly drawn on the fingertip. Some preliminary measurements were conducted that compared letter recognition using letters divided into 6 parts with letter recognition using letters divided into 12 parts. At comparable display times, there appeared to be no difference in the recognizability of letters divided into 12 or 6 parts. Because each part requires at least $4.3 \mathrm{msec}$ to generate, and therefore a greater number of parts would require longer letter-production times, it was decided to continue to divide letters into 6 parts.

Each of the four modes was tested at several different durations: St at 4.3, 26, 100, and $400 \mathrm{msec}$; Sc at 26, 100 , and $400 \mathrm{msec}$; SS at 8.6, 52, 100, and $400 \mathrm{msec}$; and $\mathrm{CS}$ at 52,100 , and $400 \mathrm{msec}$. Trials were grouped in blocks of $\mathbf{4 0}$ trials. Each block tested a particular mode and duration. The order of presentation of the modes and the order of presentation of the various durations within each mode were varied randomly. All durations within a particular mode were tested before another mode was selected for testing. A typical experimental session lasted $50 \mathrm{~min}$.

In the second set of measurements, the same four modes were tested with another group of subjects at somewhat different durations, and the slit in the SS mode was narrowed to a single column. In addition, a fifth mode was tested, discontinuous sequential (DS) mode. This mode is identical to the CS mode except that each element of the letter is turned off before the next element comes on. Preliminary measurements had indicated that the DS mode produced poorer performance than the CS mode; however, the DS bears a greater similarity to the mode tested by Beauchamp, Matheson, and Scadden (1971), and thus, it was felt, should be tested formally. The St mode was tested at $4.3,26,100$, and $400 \mathrm{msec}$, the Sc mode at 26,100 , and $400 \mathrm{msec}$, the CS mode at 
26,100 , and $400 \mathrm{msec}$, and the DS mode at $4.3,26,100$, and $400 \mathrm{msec}$. The remainder of the procedure followed that used in the first set of measurements.

Previous results with the St and Sc modes (Craig, 1980) and preliminary results with the remaining three modes had shown that there was little or no improvement in performance as duration increased beyond $400 \mathrm{msec}$. In fact, those results were the major reason the main sets of measurements were not extended beyond $400 \mathrm{msec}$. However, because the measurements made by Loomis were made at durations ranging from 1 to $1.5 \mathrm{sec}$, it was considered necessary to examine somewhat longer times and compare performances across modes. Three subjects, one who had participated in the first set of measurements and two who had participated in the second set of measurements, were tested at durations of 400 and $1,000 \mathrm{msec}$ in all five modes. The modes were presented in random order with both durations for a particular mode presented before the next mode was tested.

\section{Specifying Display Time}

An important question arises about how pattern duration or display time is specified for each mode, particularly, as in the present experiments, when the intention is to compare performance with different modes at comparable durations. There is no problem in defining the duration for patterns presented in the static mode. All the elements in the pattern are on for the same duration. However, there is a problem in specifying the duration for the other modes, because the durations for which the elements of the pattern are presented are different from the total duration the pattern is on. For example, in the DS mode, each element is activated for one-sixth of the duration of the whole pattern.

Although other definitions might be possible, it seems clear that there are two main ways of defining display time: One is the time an element of the pattern is on, and the other is the total time the pattern is on, from the onset of the first element to the offset of the last element. Both of these definitions reflect a theoretical position on how cutaneous patterns are processed. The definition used in the present experiment stressed element rather than total pattern duration. Specifically, display time was defined as the maximum time any element of the pattern was on. For four of five modes, all elements were on for the same duration. Only in the case of the CS mode were elements on for varying durations; that is, the first element to come on remained on the longest time, whereas the last element to come on remained on for the shortest time. The definition of display time used in the present study has been used previously (Craig, 1980; Taenzer, 1980). It reflects an applied bias in that display time, by this definition, is the time required before a second pattern may be generated on the array, a definition that comes from the use of the Optacon in the scan mode as a reading aid. An analysis of the data will suggest that defining display time in terms of element duration produces an ordering of the data that is consistent with certain predictions coming from measurements of temporal integration. The definition was applied to each of the modes as follows:

Static mode-Display time equaled the time the elements were on. Minimum duration possible was $4.3 \mathrm{msec}^{1}$

Scan mode-Display time equaled the time beginning with the left side of the pattern's entering on the right side of the array and ending with the left side of the pattern's exiting the left side of the array. Because all the letters, with the exception of " $I$ " and "J," occupied all six columns when centered on the array, this definition also was effectively equal to the time required before another pattern could be presented. Minimum duration possible was $4.3 \mathrm{msec} \times 6$ (columns), or $26 \mathrm{msec}$.

Slit-scan mode-In the case of a one-column slit, the elements were activated for one-sixth of the time measured from the onset of the first column to the offset of the sixth column. In the case of a two-column slit, the elements were activated for one-third the total time parts of the pattern were on. Minimum duration possible in the one-column mode was $4.3 \mathrm{msec}$ and in the twocolumn mode, $8.6 \mathrm{msec}$.
Discontinuous sequential mode-Because the letters were divided into six elements, each element was activated for one-sixth the total duration the pattern was on. Minimum duration was $4.3 \mathrm{msec}$.

Continuous sequential mode-Display time equaled the duration of activation of the first element of the pattern. In the CS mode, the first element remained on until the last element of the letter had come on and then all elements were turned off simultaneously. Minimum duration possible was $4.3 \mathrm{msec} \times 6$ (the number of parts), or $26 \mathrm{msec}$.

\section{RESULTS}

The results from the first set of measurements are shown in Figure 1. Each of the four subjects was tested for 200 trials at each duration in each mode. Thus, each point in Figure 1 represents the mean of 800 trials, with the exception of the point at $4.3 \mathrm{msec}$ in the static mode. Because of a problem with the equipment, a letter was not presented on 80 trials in that condition, and thus the point represents only 720 trials. The standard error of the mean, computed for each point by averaging across the 40-trial blocks, ranged from $2 \%$ to $4 \%$ and did not vary systematically as a function of duration or mode.

The results from the second set of measurements are shown in Figure 2. Each of the four subjects was tested for 280 trials at each duration in each mode. Each point in Figure 2 represents the mean of 1,120 trials. The standard error of the mean, computed as in the first set of measurements by averaging across 40-trial blocks, ranged from $2 \%$ to $3 \%$. The standard errors did not vary systematically as a function of duration or mode.

The results in Figures 1 and 2 were examined to see to what extent the subjects were showing practice ef-

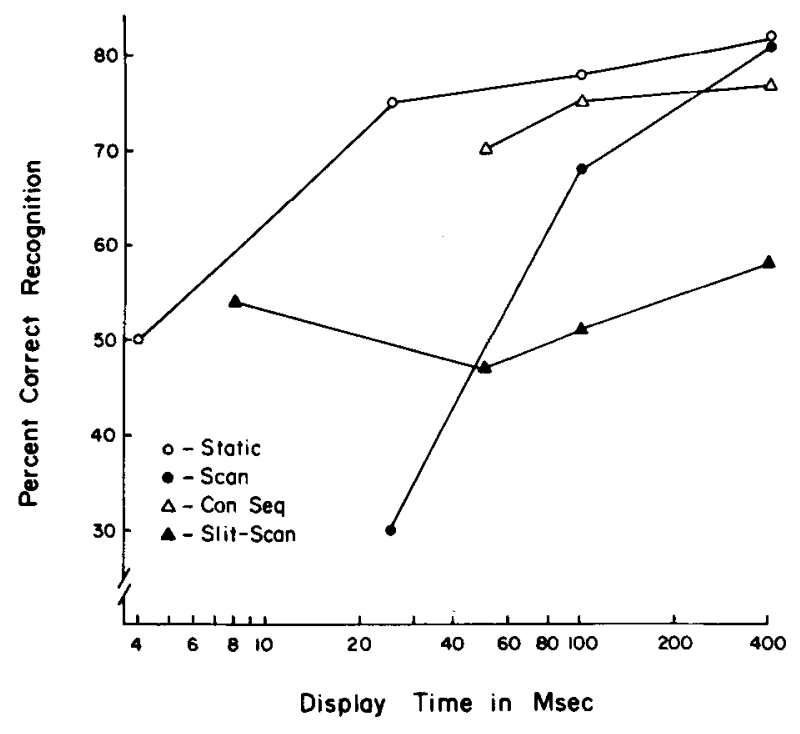

Figure 1. Percentage correct letter recognition as a function of display time in milliseconds. The results from four modes of presentation are shown. 


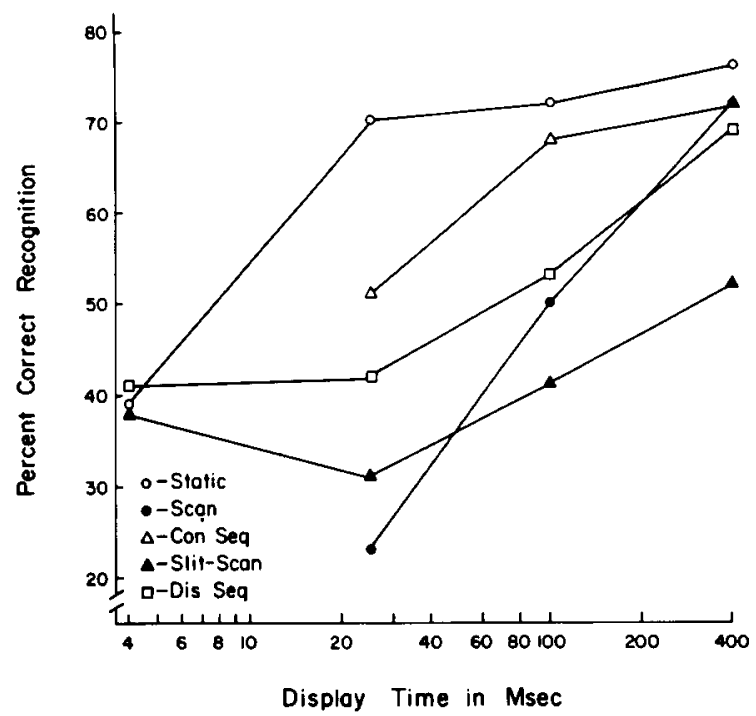

Figure 2. Percentage correct letter recognition as a function of display time in milliseconds. The results from five modes of presentation are shown.

fects. The mean percent correct for the first 80 trials averaged across subjects and durations was computed for each mode and compared with the same statistic computed for the last 80 trials. Performance in all four modes (Figure 1) showed a slight improvement, ranging from a low of $2 \%$ in the SS mode to a high of $5 \%$ in the Sc mode.

Some improvement was also seen for the results presented in Figure 2. The improvement ranged from $3 \%$ to $11 \%$. The results were further analyzed to compare the last 80 trials with the next to the last 80 trials. This comparison showed only a modest in-

Table 1

Percent Correct Recognition at Longer Durations

\begin{tabular}{lcc}
\hline & \multicolumn{2}{c}{ Duration (in Milliseconds) } \\
\cline { 2 - 3 } \multicolumn{1}{c}{ Mode } & 400 & 1000 \\
\hline Static & .85 & .88 \\
Scan & .85 & .90 \\
Continuous Sequential & .91 & .91 \\
Discontinuous Sequential & .82 & .81 \\
Slit Scan & .56 & .58 \\
\hline
\end{tabular}

Table 2

Correlations Between Modes

\begin{tabular}{lcccc}
\hline & \multicolumn{4}{c}{ Mode } \\
\cline { 2 - 5 } Mode & Scan & CS & DS & Slit Scan \\
\hline Static & .71 & .92 & .66 & .82 \\
Scan & & .56 & .65 & .76 \\
CS & & & .59 & .76 \\
DS & & & & .75 \\
\hline
\end{tabular}

Note $-C S=$ continuous sequential $; D S=$ discontinuous sequential. crease in performance, an average of $3 \%$, with the $\mathrm{Sc}$ mode showing the largest increase in this comparison, $7 \%$. It appears that additional practice might raise the overall levels of performance somewhat. However, there is no evidence that the shape of the functions shown in Figures 1 and 2 would be altered by additional practice or that the rank ordering of modes would be affected. A further indication that practice effects are not an important consideration for at least two of the modes, Sc and St, may be seen in Craig (1980). The functions for the Sc and St modes (Craig, 1980) are quite similar to those obtained in the present study, even though the previous functions were obtained from subjects with considerably more experience.

The results from the measurements made at longer durations are presented in Table 1 , with each entry representing the results of 840 trials, 280 from each of three subjects.

Stimulus-response confusion matrices were constructed for each mode in Figures 1 and 2, and the product-moment correlation was computed between modes based on the percentage of correct responses. Table 2 shows the resulting correlation coefficients from the data in Figure 2. All correlations are significant at the .01 level. Because the data shown in Figure 1 were less complete than the data in Figure 2, only the correlations computed on the latter results are presented in tabular form.

\section{DISCUSSION}

The discussion deals with three major topics: the effect of changing duration on the various modes of pattern generation, the extent to which Loomis's model of pattern recognition can account for the results, and some alternative views of pattern recognition.

The results presented in Figures 1 and 2 clearly demonstrate that increasing duration produces better letter recognition for all modes, and that the effect of duration is not uniform across modes. For example, as duration is increased from 26 to $400 \mathrm{msec}$, the Sc mode shows a change of nearly $50 \%$, whereas the St mode changes by less than $10 \%$. The interaction of mode and duration means that it is not possible to specify which one of several modes would be the most effective for presenting letters without also specifying at what duration the letters were to be presented.

The interaction of mode and duration also makes it unlikely that a single principle of information processing will be sufficient to explain why increasing duration leads to better recognition in every mode. In fact, evidence has already been produced (Craig, 1980) that the reason increasing duration in the $\mathrm{St}$ mode produces better letter recognition is that such increases result in greater perceived intensity. It was 
demonstrated that letters presented at two different durations would be equally recognizable when set to be equal in perceived intensity, an equality achieved by decreasing the amplitude of vibration at the longer duration. In the Sc mode, compensating for changes in duration by changing the amplitude of vibration did not produce equal recognizability of the patterns (Craig, 1980). The question remains, for the Sc mode and the other three modes shown in Figures 1 and 2, as to why increasing duration produces better letter recognition. Increasing intensity is unlikely to be the whole story, inasmuch as there is a clear increase in intensity as pattern duration increases from 8.6 to $100 \mathrm{msec}$ in the SS mode (Figure 1) and from 4.3 to $26 \mathrm{msec}$ in the DS and SS modes (Figure 2) without any improvement in percent correct recognition. These data introduce a complication in that they raise the question of why changing duration for DS and SS modes at some durations improves letter recognition and at other durations does not, an issue that will be considered later.

One of the primary questions this research was designed to answer was the extent to which Loomis's theory would account for the results. As noted before, Loomis's results with the TVSS showed that the SS mode with a stationary letter produced the best performance, followed by the SS mode with a moving letter; the Sc and St modes produced the poorest performance. His theory, designed to accommodate these results, also predicts that a sequential mode, which he did not test, would produce the best performance. The results shown in Figures 1 and 2 are clearly in disagreement with Loomis's results and with his theory of form perception. At all durations tested, the St mode was as good as or superior to all other modes tested. Moreover, the rank ordering of the modes changed as duration was changed. Most notably, the ranking of the Sc mode moves from producing the poorest performance at $26 \mathrm{msec}$ to producing levels of performance very nearly equal to the other modes at $\mathbf{4 0 0} \mathrm{msec}$. The fact that duration has an effect on the relative rankings of the modes is troublesome for Loomis's theory. First, there is no particular reason why duration should affect the spatial resolution of the skin. Second, the whole discussion of relative rankings is complicated considerably if such rankings are dependent upon the duration at which they are made.

Because the present results are not consistent with previous results obtained with the TVSS, it is necessary to ask what factors led to the difference in the pattern of results. One possibility is, of course, the duration of the patterns. As duration increases, the differences among the modes decrease, and it had been speculated that at longer durations the pattern of results obtained by Loomis might be seen (Craig, 1980). The results shown in Table 1 do not support this speculation, since the rankings among modes were not the same as those found by Loomis. The results do support the preliminary observations mentioned above, viz., there is little change in performance beyond $400 \mathrm{msec}$. The four modes that converge at $400 \mathrm{msec}$ in Figure 2 produce fairly similar levels of performance at $1,000 \mathrm{msec}$, with the SS mode below the other four.

Another reason for the difference between the present and earlier results is suggested in the study by Apkarian-Stielau \& Loomis (1975) in which tactile and visual form perception were compared. It was predicted in that study that blurred letters presented visually in the St mode would be harder to recognize than the same letters presented in a SS mode. The results showed no difference between the two modes. The finding that the SS mode was not superior to the St mode was attributed to the greater amount of exposure subjects had had to visually presented static letters in their lifetimes. However, it is unlikely that such an explanation would account for the present pattern of results. Although it is true that the subjects had had some previous experience with the St mode, practice effects seen with the other modes were also seen in the St mode. Also, the subjects had nearly equal exposure to the other four modes. Even with the static results removed, the pattern of the remaining results is still not consistent with Loomis's theory. Finally, the amount of experience that subjects had had with letters presented tactually in the St mode prior to these measurements is brief relative to the lifetime of experience with letters presented visually in the St mode, the explanation that Loomis proposed.

Another possibility that might account for the differences between the present results and Loomis's results is the differences in the two types of display. The display used by Loomis was relatively large, $25 \times 25 \mathrm{~cm}$, and in contact with the back. The Optacon array is considerably smaller and in contact with a single finger. These two factors, size and location of stimulation, might produce some differences in the way letters are processed. The letters used by Loomis were about 10 times the size of the letters used in the present study, yet the change in spatial sensitivity between the back and finger may be even greater than a factor of 10 . For example, the twopoint limen is about 15 times larger on the back than it is on the index fingertip (Weinstein, 1968). It is possible that low-pass spatial filtering played much more of a role in Loomis's study than in the present study because a greater proportion of the letters he employed exceeded the low-pass cutaneous filter.

A recent study by Loomis (1980) would suggest that this is indeed the case. Using the Optacon and letters of different sizes, Loomis found that small letters were more recognizable presented in a slit scan 
mode than when they were presented in a static mode. For larger letters, of the same size employed in the present study, the static mode produced slightly better performance than the slit scan mode. Pattern durations were long, $1.5 \mathrm{sec}$, a condition that would tend to reduce the differences among modes of presentation. If the patterns used in the present study are large enough for Loomis's limited spatial resolution hypothesis not to apply, the question still remains as to why the various modes produced the relative levels of performance that they did.

At the briefest time, $4 \mathrm{msec}$, Figure 2 shows that three modes, St, SS, and DS, produce similar levels of performance. Some recent measurements in our laboratory suggest that with very brief intervals between pattern elements, approximately $12 \mathrm{msec}$ and less, the skin is capable of nearly complete temporal integration. Applied to the present results, such a finding indicates that it should make little difference whether or not all the elements of a pattern are presented simultaneously, as in the St mode, or sequentially over time, as in the SS and DS modes, if patterns are presented in a brief enough period of time. The data obtained at $4 \mathrm{msec}$ for three modes were examined, and the product-moment correlations on the percentage of correct responses for the 26 letters were computed. The correlation between St and DS was .92, between St and SS, .89, and between DS and SS, .92. As Figure 2 shows, at $26 \mathrm{msec}$ the performance levels are quite different, and the correlations based on correct responses have dropped to a mean of .67. Even at $400 \mathrm{msec}$, when the performance levels for the St and DS modes are similar, the correlation has dropped further, to .07 . Taken together, these results suggest that at brief times vibrotactile patterns are processed in a similar fashion regardless of mode; but as display time lengthens, the manner of processing patterns may change. What the nature of these changes might be will be considered below.

The fact that the three modes produce similar results at $4 \mathrm{msec}$ reflects on an issue raised earlier, viz., the definition of display time. If display time were defined as the total time required to generate the pattern, that is, from the onset of the first element to the offset of the last, then both the DS and SS functions would be shifted to the right by a factor of six, and the minimum duration for these two modes would be $26 \mathrm{msec}$ rather than $4 \mathrm{msec}$. This would mean that at the first "comparable" duration, $26 \mathrm{msec}$, performance in the St mode would be $30 \%$ higher than performance in the DS and SS modes. Defining display times as we have in the present study brings the three functions together, a result that lends support to the particular definition of display time we have used.

There are some indications that patterns presented in the CS mode are also processed in a manner sim- ilar to the way they are processed in the St mode. First, the overall performance level in the CS mode is higher than in the DS, SS, and Sc modes, with the level of performance approaching that of the St mode. Second, the highest correlation between any two modes shown in Table 2 is between the CS and St modes.

A further experimental manipulation that might reveal the extent to which the St, SS, and DS modes are processed in a similar fashion at brief durations and the St and CS modes are processed similarly at all durations would require varying the intensity of vibration. The basis for this manipulation rests on the observation, as noted before, that increasing the intensity of a vibration increases recognizability for $\mathrm{St}$ patterns but not for Sc patterns (Craig, 1980). It should be that increasing perceived intensity (by increasing the amplitude of vibration) for SS and DS patterns will improve recognizability at brief durations but not at long durations. Turning to the CS mode, if it is being processed in a fashion similar to that of the St mode, then the reason the CS mode shows improved recognizability with increasing duration is that perceived intensity is increasing. Patterns presented in the CS mode for different durations could be equated for perceived intensity by increasing the amplitude of vibration of the briefer patterns. If letters were equally recognizable when presented for different durations, equated for perceived intensity, this would be evidence, first, that increasing duration produced better performance due to increasing intensity and, second, that the CS and St modes were being processed in a similar fashion.

The suggestion that, at the briefest duration, the CS, DS, and SS modes are processed in a similar fashion because of temporal integration carries with it the implication that the patterns are processed in a parallel fashion. As display time increases, there may be a shift away from parallel towards serial processing for the DS and SS modes. On the functions shown in Figure 2, the only points at which increasing duration fails to improve performance are between 4 and $26 \mathrm{msec}$ for the SS and DS modes. Our measurements suggest that durations beyond $26 \mathrm{msec}$ are beginning to exceed the limits of complete temporal integration, with the result that patterns generated in these two modes can no longer be processed in parallel but would have to be processed serially. Presumably, performance improves for these two modes as display time increases beyond $26 \mathrm{msec}$ because attention can now be switched from one part of the letter to another as the pattern is generated, allowing salient elements to be extracted more easily from the total pattern.

It should be pointed out that the elements in the CS mode are available for parallel processing. That is, all elements are on simultaneously for a period of time 
equal to one-sixth of the display times shown in Figures 1 and 2 for this mode. If further work should strengthen the contention that the CS mode is being processed if a parallel fashion, it might be more appropriate in the future to redefine display time for the CS mode and to shift the CS function to the left, reducing the display times by a factor of six.

In analyzing the results in terms of the serial-parallel processing distinction, the same line of reasoning applied to the CS mode could be applied to the Sc mode, viz., that there is a brief temporal interval, one-sixth the total duration, when the whole pattern is centered on the display and can be processed in a parallel fashion. There are difficulties with this interpretation, however. The level of performance in the Sc mode is considerably below that of the CS mode at the same display times. Even if one were to consider the display time for the Sc mode to be one-sixth of that shown in Figures 1 and 2 and shifted the Sc functions to the left (following the same line of reasoning applied to the CS mode), performance levels would still be considerably below those achieved in the St mode. In addition, the correlation between the $\mathrm{Sc}$ and $\mathrm{St}$ modes shown in Table 2 is not particularly high.

Although the present results suggest that a serialparallel processing framework may be useful in viewing the data, it is not surprising that such a framework cannot easily account for all the changes in pattern recognition that occur as a function of display time or mode of generation. There are a number of additional factors known to affect cutaneous pattern recognition, such as forward and backward masking (Craig, 1976, 1978, 1980), communality (Geldard \& Sherrick, 1965; Gottheil, Cholewiak, \& Sherrick, 1978), and spatial masking (Gilson, 1969; Loomis \& Apkarian-Stielau, 1976). It is likely that the extent to which these factors will affect pattern recognition depends, as much as does the serial-parallel processing distinction, upon both the display time and the particular mode of pattern generation.

\section{REFERENCES}

Apkarian-Stielau, P., \& Loomis, J. M. A comparison of tactile and blurred visual form perception. Perception \& Psychophysics, $1975,18,362-368$.
BACH-Y-RITA, P. Brain mechanisms in sensory substitution. New York: Academic Press, 1972.

Beauchamp, K. L., Matheson, D. W., \& Scadden, L. A. Effects of stimulus-change method on tactile-image recognition. Perceptual and Motor Skills, 1971, 33, 1067-1070.

Craig, J. C. Vibrotactile letter recognition: The effects of a masking stimulus. Perception \& Psychophysics, 1976, 20, 317-326.

Craig, J. C. Vibrotactile pattern perception: Extraordinary observers. Science, 1977, 196, 450-452.

Craig, J. C. Vibrotactile pattern recognition and masking. In G. Gordon (Ed.), Active touch-The mechanism of recognition of objects by manipulation: A multi-disciplinary approach. Oxford: Pergamon Press, 1978.

Craig, J. C. Modes of vibrotactile pattern perception. Journal of Experimental Psychology: Human Perception and Performance, $1980,6,151-166$

Geldard, F. A., \& Sherrick, C. E. Multiple cutaneous stimulation: The discrimination of vibratory patterns. Journal of the Acoustical Society of America, 1965, 37, 797-801.

Gilson, R. D. Vibrotactile masking: Some spatial and temporal aspects. Perception \& Psychophysics, 1969, 5, 176-180.

Goldish, L. H., \& TAYLOR, H. E. The Optacon: A valuable device for blind persons. New Outlook for the Blind, 1974, 68, 49-56.

Gottheil, E. F., Cholewiak, R. W., \& Sherrick, C. E. The discrimination of vibratory patterns on a tactile matrix. Bulletin of the Psychonomic Society, 1978, 11, 21-24.

Loomis, J. M. Tactile letter recognition under different modes of stimulus presentation. Perception \& Psychophysics, 1974, 16, 401-408.

Loomis, J. M., \& Apkarian-Stielau, P. A lateral masking effect in tactile and blurred visual letter recognition. Perception \& Psychophysics, 1976, 20, 221-226.

Loomis, J. M. Interaction of display mode and character size in vibrotactile letter recognition. Bulletin of the Psychonomic Society, 1980, 16, 385-387.

Sherrick, C. E. Cutaneous communication. In W. D. Neff (Ed.), Contributions to sensory physiology. New York: Academic Press, 1979.

TAEnze R, J. C. Visual word reading. IEEE Transactions on ManMachine Systems, 1970, MMS-11, 44-53.

WEINSTEIN, $S$. Intensive and extensive aspects of tactile sensitivity as a function of body part, sex, and laterality. In D. R. Kenshalo (Ed.), The skin senses. Springfield, Ill: Thomas, 1968.

White, B. W., Saunders, F. A., Scadden, L., Bach-y-Rita, P., \& Collins, C. C. Seeing with the skin. Perception \& Psychophysics, 1970, 7, 23-27.

\section{NOTE}

1. Because of the way in which the tactile display is designed and interfaced, a 4.3-msec electrical signal may produce mechanical vibration that lasts several milliseconds longer than $4.3 \mathrm{msec}$.

(Manuscript received July 17, 1980; revision accepted August 31,1981 .) 